\title{
Scalable Dissipative Preparation of Many-Body Entanglement
}

\author{
Florentin Reiter, ${ }^{1, *}$ David Reeb, ${ }^{2}$ and Anders S. Sørensen ${ }^{1}$ \\ ${ }^{1}$ Niels Bohr Institute, University of Copenhagen, Blegdamsvej 17, DK-2100 Copenhagen, Denmark \\ ${ }^{2}$ Institute for Theoretical Physics, Leibniz Universität Hannover, Appelstrasse 2, 30167 Hannover, Germany \\ (Received 4 March 2015; revised manuscript received 5 August 2015; published 20 July 2016)
}

\begin{abstract}
We present a technique for the dissipative preparation of highly entangled multiparticle states of atoms coupled to common oscillator modes. By combining local spontaneous emission with coherent couplings, we engineer many-body dissipation that drives the system from an arbitrary initial state into a Greenberger-HorneZeilinger state. We demonstrate that using our technique highly entangled steady states can be prepared efficiently in a time that scales polynomially with the system size. Our protocol assumes generic couplings and will thus enable the dissipative production of multiparticle entanglement in a wide range of physical systems. As an example, we demonstrate the feasibility of our scheme in state-of-the-art trapped-ion systems.
\end{abstract}

DOI: 10.1103/PhysRevLett.117.040501

Multiparticle entanglement is an essential resource for quantum computation and information [1], e.g., in quantum error correction [2,3], quantum memories [4], and entanglement-enhanced quantum measurement schemes $[5,6]$. Among the most important states that exhibit genuine multiparticle entanglement are Greenberger-HorneZeilinger (GHZ) states:

$$
|\mathrm{GHZ}\rangle=\frac{1}{\sqrt{2}}(|000 \ldots 0\rangle+|111 \ldots 1\rangle)
$$

Deterministic preparation of such states has so far been performed using time-dependent unitary gates [7-10], which have recently yielded impressive progress towards entangling larger numbers of qubits [11-13], and feedback control schemes [14-16]. These operations, however, suffer from quantum noise, causing decoherence and dissipation so that it remains difficult to prepare high-fidelity multiparticle entangled states with these methods [17,18]. Recently, dissipative state preparation has been proposed as an alternative approach where the dissipative environment is actively engineered and used to prepare states relevant for quantum information and simulation [19-23]. Numerous theoretical studies on the production of bipartite entangled states [24-31] have since been performed and the first experimental demonstrations [32-35] have been realized. More recently, dissipative schemes for the generation of multipartite entangled states [36-51] have also become available, e.g., for the preparation of states stabilized by local interactions $[20,23,42]$. It has, however, remained a challenge to prepare in a scalable way states, like GHZ, that are highly entangled in the sense that they cannot be stabilized by local operators $[21,23,40]$.

In this Letter, we extend the range of the dissipative approach by demonstrating a scalable technique for the dissipative preparation of highly entangled states of many particles. We show that, by using local spontaneous emission as a generic source of dissipation, we can engineer nontrivial many-body dissipative interactions [48] which are tailored to produce multiparticle GHZ states. Our scheme is deterministic and operates by continuous optical driving from an arbitrary initial state towards the desired steady state using weak classical fields. The preparation time of our protocol is found to exhibit a favorable polynomial scaling with the number of qubits. In addition to our generic system-independent scheme, we describe an implementation in a system of trapped ions.

In our protocol, preparation of a steady GHZ state Eq. (1) of $N$ qubits starting from an arbitrary initial state is accomplished by two simultaneous operations shown in Fig. 1: (i) pumping all states with more than zero but less than all qubits in state $|1\rangle\left(0<n_{1}<N\right)$ to the state $|0\rangle^{\otimes N}$, which can be written as a superposition $|0\rangle^{\otimes N}=$ $|\mathrm{GHZ}\rangle+\left|\mathrm{GHZ}_{-}\right\rangle$, and (ii) removing the GHZ state with the wrong phase, $\left|\mathrm{GHZ}_{-}\right\rangle=\left(|0\rangle^{\otimes N}-|1\rangle^{\otimes N}\right) / \sqrt{2}$, from the subspace spanned by $|0\rangle^{\otimes N}$ and $|1\rangle^{\otimes N}$. Operation (i) is implemented such that it fulfills the main requirement of a dissipative protocol: it has to pump an exponential number of states efficiently, i.e., in polynomial time. In principle, standard optical pumping [52] satisfies this criterion as well, but would also erase the state $|1\rangle^{\otimes N}$, thus ruling out

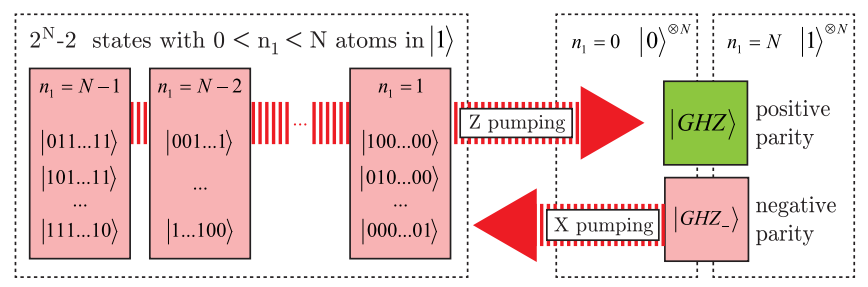

FIG. 1. Protocol. Preparation of a GHZ state of $N$ qubits is realized by two operations: (i) " $Z$ pumping" of states with other than 0 or $N$ atoms in state $|1\rangle$ to $|0\rangle^{\otimes N}$ and (ii) $|0\rangle^{\otimes N}$ is a superposition of $|\mathrm{GHZ}\rangle$ and $\left|\mathrm{GHZ}_{-}\right\rangle=\left(|0\rangle^{\otimes N}-|1\rangle^{\otimes N}\right) / \sqrt{2}$, so that $\left|\mathrm{GHZ}_{-}\right\rangle$needs to be removed by the parity-selective "X pumping." 
the possibility to prepare $|\mathrm{GHZ}\rangle$ with high fidelity. Instead, we design a new procedure that is selective in the number of atoms in $|1\rangle$. This allows us to pump only states with $0<n_{1}<N$ to states with $n_{1}-1$, and thus eventually to $|0\rangle^{\otimes N}\left(n_{1}=0\right)$. We refer to this operation shown in Fig. 1 as " $Z$ pumping," since it is based on counting the number of atoms in the eigenbasis of $Z=|0\rangle\langle 0|-| 1\rangle\langle 1|$.

A second operation (ii) is required to remove the undesired $\left|\mathrm{GHZ}_{-}\right\rangle$state in a continuous manner, as also illustrated in Fig. 1. To this end, we perform a pumping process selective in the parity $\mathcal{P}=\prod_{a=1}^{N} X_{a}\left(X_{a}=|1\rangle_{a}\langle 0|+\right.$ $\left.|0\rangle_{a}\langle 1|\right)$. Here, we apply the recipe for stabilizer pumping from Ref. [40] to the case of the parity stabilizer $\mathcal{P}$ : Expressed in terms of the eigenstates of $X,| \pm\rangle=\left(|0\rangle_{ \pm}\right.$ $|1\rangle) / \sqrt{2}$, any state $|\psi\rangle$ with $\mathcal{P}|\psi\rangle=+1|\psi\rangle$, such as $|\mathrm{GHZ}\rangle$, is a superposition of only those product states that contain an even number $n_{-}$of $|-\rangle$qubits, e.g., for $N=3$, $|\mathrm{GHZ}\rangle=(|+++\rangle+|+--\rangle+|-+-\rangle+|--+\rangle) / 2$. On the other hand, $n_{-}$is odd for any state $|\psi\rangle$ with $\mathcal{P}|\psi\rangle=-1|\psi\rangle$, such as $\left|\mathrm{GHZ}_{-}\right\rangle$, for $N=3,\left|\mathrm{GHZ}_{-}\right\rangle=$ $(|++-\rangle+|+-+\rangle+|-++\rangle+|---\rangle) / 2$. By pumping all states with odd $n_{-}$to other states, in the following referred to as " $X$ pumping," we achieve the depumping of $\left|\mathrm{GHZ}_{-}\right\rangle$.

The two operations required for our protocol can be realized using a generic setup as described next. We assume a general system of $N$ particles ("atoms"), shown in Figs. 2(a)-2(c). Each atom supports two stable ground states $|0\rangle$ and $|1\rangle$ and two excited states $|e\rangle$ and $|f\rangle$. The atoms are driven by classical multitone driving fields, with identical amplitudes on all ions, as described by the Hamiltonians

$$
\begin{gathered}
H_{\text {drive }, Z}^{(F)}=\frac{1}{2} \Omega_{Z}^{(F)} e^{i \Delta_{Z}^{(F)} t} \sum_{a=1}^{N}|e\rangle_{a}\langle 1|+\text { H.c. }, \\
H_{\text {drive }, X}^{(F)}=\frac{1}{2} \Omega_{X}^{(F)} e^{i \Delta_{X}^{(F)} t} \sum_{a=1}^{N}|f\rangle_{a}\langle-|+\text { H.c., }
\end{gathered}
$$

with strengths $\Omega_{l}^{(F)}$ and detunings $\Delta_{l}^{(F)}$, where $l=Z, X$ denotes the desired operation and $F$ the field tone. The transitions of the atoms are collectively coupled to two harmonic oscillator modes, $b$ and $c$, e.g., two resolved resonator modes in cavity or circuit QED [7,10], or two phononic modes in an ion trap setup [9,11-13], as modeled by the Hamiltonians

$$
\begin{aligned}
& H_{\mathrm{int}, Z}=g b^{\dagger} \sum_{a=1}^{N}|1\rangle_{a}\langle e|+\text { H.c., } \\
& H_{\mathrm{int}, X}=g c^{\dagger} \sum_{a=1}^{N}|-\rangle_{a}\langle f|+\text { H.c., }
\end{aligned}
$$

with $g$ being the coupling constant. For the dissipative process we consider decay by spontaneous emission from the excited states $j$ to the ground states $i$ at a rate $\gamma_{i j}$
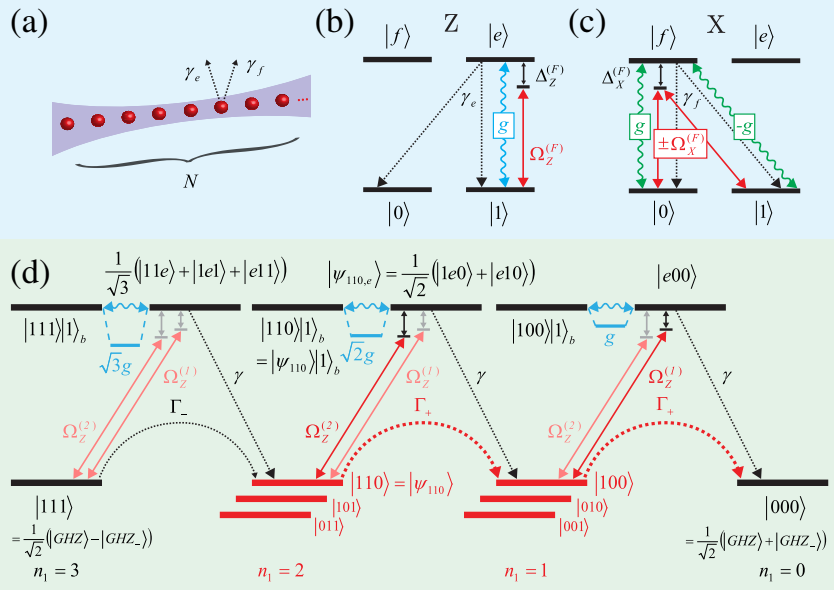

FIG. 2. Setup (a)-(c) and dissipative mechanism (d) for GHZ preparation, shown for $N=3$ qubits. Setup (a): We consider a chain of $N$ subsystems ("atoms") with four levels, coupled to two common harmonic oscillators. As dissipative processes, we assume spontaneous emission from the excited levels $\gamma_{e / f}$. We apply coupling configurations $Z$ (b) and $X$ (c) consisting of $2(N-1)$ driving tones $\Omega_{Z}^{(F)}$ with detunings $\Delta_{Z}^{(F)}$ in (b), $2\lfloor(N+$ 1)/2」 tones with $\Omega_{X}^{(F)}$ and $\Delta_{X}^{(F)}$ in (c), and couplings of the atoms to the oscillator modes $g$. (d) $Z$ pumping towards $|0\rangle^{\otimes N}$ using the couplings in (b). Ground states are coupled to atom-excited states by weak driving. Depending on the number $n_{1}$ of atoms in $|1\rangle$, the excited states form dressed states with oscillator-excited states at energies $\pm \sqrt{n_{1}} g$. By applying fields with detunings $\left|\Delta_{Z}^{(F)}\right|=$ $\sqrt{F} g$ for $1 \leq F \leq N-1$ (only the red-detuned fields are shown), all states except $|\mathrm{GHZ}\rangle$ and $\left|\mathrm{GHZ}_{-}\right\rangle$are pumped to $|0\rangle^{\otimes N}=\left(|\mathrm{GHZ}\rangle+\left|\mathrm{GHZ}_{-}\right\rangle\right) / \sqrt{2}$. $\left|\mathrm{GHZ}_{-}\right\rangle$is emptied by the parity-selective $X$ pumping as described in the text.

(with $\gamma_{j}=\sum_{i} \gamma_{i j}$ ), described by jump operators $L_{\gamma_{i j}, a}=$ $\sqrt{\gamma_{i j}}|i\rangle_{a}\langle j|$ for $i \in\{0,1\}, j \in\{e, f\}$ acting incoherently on each atom $a$.

We realize the $Z$ - and $X$-pumping operations by engineering selected transitions to be driven resonantly, while suppressing others due to off-resonant driving. For $Z$ pumping we use the coupling configuration in Fig. 2(b). Here, a coupling $g$ of the oscillator $b$ to the transition $|e\rangle \leftrightarrow|1\rangle$ and a weak drive on the same transition are used to effectively "count" the number of atoms in state $|1\rangle$. In Fig. 2(d), we illustrate this mechanism for $N=3$ qubits. The weak driving tones $\Omega_{Z}^{(F)}$ couple the ground states to atom-excited states. For example, we consider $\left|\psi_{110}\right\rangle=|110\rangle$, which is coupled to $\left|\psi_{110, e}\right\rangle=(|e 10\rangle+$ $|1 e 0\rangle) / \sqrt{2}$. This state is in turn coupled to an oscillatorexcited state $\left|\psi_{110}\right\rangle|1\rangle_{b}$ by the atom-oscillator coupling. Because of constructive interference between the two terms in $\left|\psi_{110, e}\right\rangle$, this coupling has a strength of $\sqrt{2} g$. As a consequence of the strong atom-oscillator coupling, the atom- and the oscillator-excited state form dressed states $\left|\psi_{110, \pm}\right\rangle=\left(\left|\psi_{110, e}\right\rangle|0\rangle_{b} \pm\left|\psi_{110}\right\rangle|1\rangle_{b}\right) / \sqrt{2}$ at detunings $\Delta_{ \pm}= \pm \sqrt{2} g$; see Fig. 2(d). Applying a weak driving field 
with a detuning $\left|\Delta_{Z}\right|=\sqrt{2} g$, one thus excites ground states with $n_{1}=2$ to excited states like $\left|\psi_{110, e}\right\rangle$. Since $|e\rangle$ is subject to spontaneous emission to $|0\rangle$ and $|1\rangle,\left|\psi_{110, e}\right\rangle$ decays either back to the manifold of states with $n_{1}=2$ or "forward" to states with $n_{1}=1$. In general, the couplings of the atom- and oscillator-excited states have a strength of $\sqrt{n_{1}} g$ that depends on the number $n_{1}$ of atoms in $|1\rangle$, so that the dressed states are shifted by $\Delta_{ \pm}= \pm \sqrt{n_{1}} g$. This creates a resonance condition depending on $n_{1}$ of the initial state, which we can use to selectively drive a manifold. Applying a drive with $\left|\Delta_{Z}\right|=\sqrt{1} g$, states with $n_{1}=1$ are thus pumped to $n_{1}=0$, and thereby to $|\mathrm{GHZ}\rangle$ and $\left|\mathrm{GHZ}_{-}\right\rangle$. On the other hand, with the detunings $\Delta_{Z}= \pm g$ and $\pm \sqrt{2} g$, the state $|1\rangle^{\otimes N}$ is excited only off resonantly and thus decays slowly [see Fig. 2(d)], so that $\mid$ GHZ $\rangle$ remains almost unaffected.

To realize the full $Z$-pumping process based on the mechanism above, we apply a weak drive consisting of $2(N-1)$ tones $\Omega_{Z}^{(F)}$ with detunings $\Delta_{Z}^{(F)}= \pm \sqrt{F} g$ ranging from $F=1$ to $F=N-1$. This gives rise to effective decay processes [53] described by

$L_{\gamma_{0}, a, Z}^{\left(n_{1}\right)}=\sqrt{\gamma_{0, Z}^{\left(n_{1}\right)}}|0\rangle_{a}\langle 1| P_{n_{1}}, \quad\left(1 \leq n_{1} \leq N-1\right)$.

Here, $P_{n_{1}}$ is the projector onto the ground states with $n_{1}$ atoms in state $|1\rangle, a$ denotes the atom subject to decay, and $\gamma_{0, Z}^{\left(n_{1}\right)}=\gamma_{0 e}\left(\Omega_{Z}^{\left(n_{1}\right)} / \gamma_{e}\right)^{2}$ are the strongly enhanced decay rates of the states that are resonantly excited. Each ground state with $1 \leq n_{1} \leq N-1$ then decays towards one with $n_{1}-1$ by the engineered spontaneous emission in Eq. (6). The concatenation of these decay processes causes a continuous drift towards states with smaller $n_{1}$, finally ending at the state $|0\rangle^{\otimes N}$ with $n_{1}=0$. For a suitable choice of the $\Omega_{Z}^{\left(n_{1}\right)}$ [53], the preparation time $\tau$ of $|0\rangle^{\otimes N}$ and the corresponding rate $\Gamma_{+}=1 / \tau$ can then have a favorable scaling $\tau \propto 1 / \Gamma_{+} \sim \log (N)$, which is similar to optical pumping where the transition rate of each level is proportional to the number $n_{1}$ of its excitations. However, as opposed to standard optical pumping, the $Z$ pumping is engineered such that it does not affect $|1\rangle^{\otimes N}$, and thus neither $|\mathrm{GHZ}\rangle$, since resonant excitation out of $|1\rangle^{\otimes N}$ would require the tone $F=N$. By excluding such tones from the drive, the excitation out of $|1\rangle^{\otimes N}$ is off resonant and thus much weaker. The weak off-resonant excitation of $|1\rangle^{\otimes N}$ by the other driving tones leads only to a small leakage from $|\mathrm{GHZ}\rangle$. Since the energy gap between the resonances and the driving tones $\left(\sqrt{N}-\sqrt{n_{1}}\right) g$ decreases with $N$, the leakage rate from $|\mathrm{GHZ}\rangle$ increases with the number of qubits, and can be estimated to be $\Gamma_{-}^{Z} \sim\left(N \gamma_{e} / g^{2}\right) \sum_{n_{1}=1}^{N-1} n_{1}\left(\Omega_{Z}^{\left(n_{1}\right)} /\left(N-n_{1}\right)\right)^{2}$. The resulting error can, however, be compensated by reducing the speed of the protocol by a small polynomial factor, as we discuss below.
Having operation (i) realized, we now turn to operation (ii), the $X$ pumping, which removes states with an odd number $n_{-}$of atoms in $|-\rangle$. To implement it we use a similar mechanism as was used to "count" $n_{1}$ above, except that now we need to do this in a different basis. We achieve this operation using the coupling configuration in Fig. 2(c): Coupling the transitions from $|0\rangle$ to $|f\rangle$ and from $|1\rangle$ to $|f\rangle$ by fields of the same strength, but with the opposite phase, results in a coupling of the transition from $|-\rangle$ to $|f\rangle$. To avoid interference with the $Z$ pumping, we consider a second excited state $|f\rangle$ and a second oscillator mode $c$. It is, nonetheless, possible to implement the described operations with a single excited level and a single oscillator mode in a stroboscopic manner, resulting in a quasisteady state.

In the $X$ pumping, the transitions between the excited level $|f\rangle$ and the ground levels $|0\rangle$ and $|1\rangle$ are coherently coupled to the harmonic oscillator $c$ and excited by a multitone drive $\Omega_{X}^{(F)}$. Opposite phases on both transitions result in a coupling of the transition $|f\rangle \leftrightarrow|-\rangle$, similar to the coupling $|e\rangle \leftrightarrow|1\rangle$ in the $Z$ configuration. In this way, we make the $X$ pumping selective in $n_{-}$in a similar manner as the $Z$ pumping is selective in $n_{1}$ : Applying $2\lfloor(N+1) / 2\rfloor$ field tones with detunings $\Delta_{X}^{(F)}= \pm \sqrt{F} g$ for $F=1,3,5, \ldots(F \leq N)$, we resonantly excite $\left|\mathrm{GHZ}_{-}\right\rangle$to dressed states which lie at $\pm \sqrt{n_{-}} g$ for $n_{-}=1,3,5, \ldots,\left(n_{-} \leq N\right)$. Thereby, we make $\left|\mathrm{GHZ}_{-}\right\rangle$ decay to random states by effective spontaneous emission with a strong rate $\Gamma_{+}^{X} \sim 2\left(\Omega_{X}^{(F)}\right)^{2} / \gamma_{f}$, Similar to the $Z$ pumping, the decreasing energy gap between the dressed states gives rise to a leakage rate from $\mathrm{GHZ}, \Gamma_{-}^{X} \sim$ $N^{2} \gamma_{f} \Omega_{X}^{2} / g^{2}$ (using $\Omega_{X}^{(F)}=\Omega_{X}$ for odd $F$ and $\Omega_{X}^{(F)}=0$ for even $F$ ), which increases with the number of qubits $N$.

The simultaneous action of the $Z$ and the $X$ pumping prepares $|\mathrm{GHZ}\rangle$ and maintains it as the unique steady state of the dissipative dynamics. However, since the $Z$ pumping is disturbed by the $X$ pumping, the latter has to be sufficiently weak so that the $Z$ pumping has a sizable probability of reaching the final state $|0\rangle^{\otimes N}$ before being subject to $X$ pumping; picking similar rates for the $X$ pumping and the total $Z$ pumping $\Gamma_{+}$, this requirement does not slow down the preparation process significantly [53]. To find the preparation time we can consider a simple model where the rate of pumping to $|0\rangle^{\otimes N}$ is determined by $\Gamma_{+}^{Z}$, and where $|\mathrm{GHZ}\rangle$ and $\left|\mathrm{GHZ}_{-}\right\rangle$are pumped out with rates $\Gamma_{-}=\Gamma_{-}^{Z}+\Gamma_{-}^{X}$ and $\Gamma_{+}^{X}$, respectively. Further details on the model are given in the Supplemental Material [53], but in short, we find that the steady state fidelity $F_{\mathrm{GHZ}}$ is determined by the ratio of the decay out due to off-resonant excitation at the rate $\Gamma_{-} \sim \gamma N \Omega^{2} / g^{2}$ and the effective preparation rate $\Gamma_{+} \sim \Omega^{2} / \gamma$ [using $\Omega_{X}^{(F)} \sim \Omega / \sqrt{N \log N}$, $\Omega_{Z}^{(F)} \sim \Omega$ for $F \leq 2 N / 3$, and $\Omega_{Z}^{(F)} \sim \sqrt{2(N-F) / F} \Omega$ for $F \geq 2 N / 3$ ]. This gives a steady state error $\mathcal{E}=\Gamma_{-} / \Gamma_{+} \sim N \gamma^{2} / g^{2}$, which is approached exponentially in time $\sim e^{-t \Gamma_{+}}$. 
To avoid having an increasing error with the qubit number $N$, we assume that we can control the decay rates of the excited states $|e\rangle$ and $|f\rangle$. This is, for instance, the case if the states are metastable states coupled to higher lying unstable states with a laser field $[33,34]$. The increase of the error with $N$ can then be compensated by having a sufficiently low decay rate $\gamma \sim g \sqrt{\mathcal{E}} / \sqrt{N}$, which keeps the error $\mathcal{E}$ constant for growing $N$, but prolongs the necessary preparation time $\tau_{\mathrm{GHZ}}$. These considerations, however, assume a weak driving $\Omega$, whereas for strong driving the pumping rate becomes limited by power broadening. We therefore need to use a suitably low driving strength $\Omega \sim \gamma / \sqrt{N}$, which sets a limit on the preparation rate. These considerations and parameter values can be turned into a rigorous upper bound on the preparation time [53]:

$$
\tau_{\mathrm{GHZ}} \propto N^{3 / 2}(\log N)\left(\log \frac{1}{\mathcal{E}}\right) /(g \sqrt{\mathcal{E}}) .
$$

We can thus prepare a GHZ state at any desired fidelity $F_{\mathrm{GHZ}}=1-\mathcal{E}$ within a preparation time that has a loworder $\left(N^{3 / 2}\right)$ polynomial scaling in the number of qubits, for a coupling $g$ independent of $N$. If instead the total preparation time is restricted to $t<T_{\max }$, then the preparation error of an $N$-particle GHZ state will necessarily obey $\mathcal{E}>N \gamma^{2} / g^{2}$ and $\mathcal{E}>e^{-T_{\max } \Gamma_{+}}$, limiting the achievable fidelity to $F_{\mathrm{GHZ}} \lesssim 1-N /\left(g T_{\max }\right)^{2}$ [53].

To confirm the results of the simple model, we simulate the protocol numerically: In Fig. 3(a) we plot the time evolution towards a steady GHZ state for $N=2, \ldots, 8$
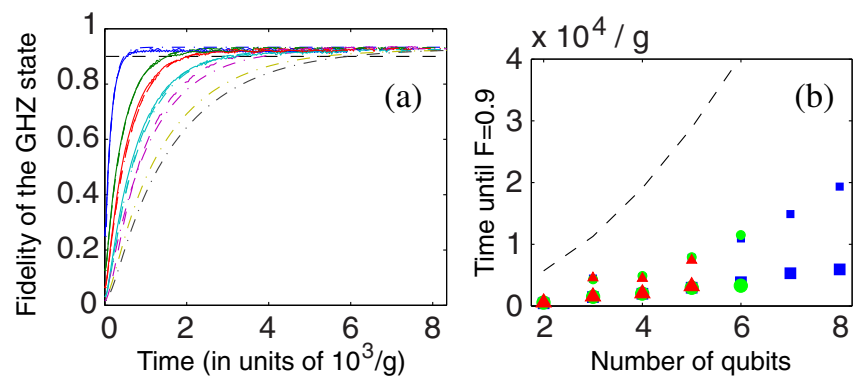

FIG. 3. Evolution towards a steady GHZ state. Starting from a fully mixed state, we numerically solve an effective master equation. The curves in (a) show the evolution for 2-8 qubits (different colors from blue to black) and are obtained by numerically optimizing all available parameters to reach a fidelity of $F_{\mathrm{GHZ}}=0.9$ (black dashed line) within as short a time as possible. In (b) we show the scaling of the preparation time with the number of qubits. Both (a) and (b) show different degrees of truncation of the Hilbert space (dash-dotted lines and blue squares, effective ground state dynamics after adiabatic elimination; solid lines and green circles, one excitation; dashed lines and red triangles, two excitations). In (b), small symbols represent analytically optimized parameters and large symbols numerically optimized parameters. We find a polynomial scaling of the preparation time that is within our analytical bound [black dashed line in (b)]. qubits resulting from our protocol. The plots are obtained by numerically simulating an effective master equation [54] as well as the complete master equation truncated to one or two excitations. Here we optimize the available parameters (driving strengths, tunable decay rates) to reach a fidelity $F_{\mathrm{GHZ}}=0.9$ of the GHZ state in minimum time. The resulting preparation times and the analytical bound from Eq. (7) is shown in Fig. 3(b). These results confirm that the scheme exhibits a low-order polynomial scaling of the preparation time with the number of qubits. In contrast to the scheme in Ref. [40], our protocol requires only two operations for a GHZ state of $N$ qubits. Furthermore, the highly directed $Z$ pumping is only weakly perturbed by the polynomially weaker $X$ pumping and thus allows for a polynomial scaling of the protocol.

The ingredients necessary for our scheme are available in trapped ion experiments. One suitable setup consists of a chain of $N$ trapped ions, each with two (meta)stable ground levels $|0\rangle$ and $|1\rangle$ and two auxiliary levels $|e\rangle$ and $|f\rangle$. Tunable decay of the auxiliary levels by spontaneous emission can be realized by a repumper to a higher lying rapidly decaying state $[33,34]$. Two phononic modes, cooled to the ground state, and coupled to the sidebands of the ions' transitions, can be used as the harmonic oscillators $b$ and $c$. For the pumping, we require $2(N-1)$ field tones in the $Z$ configuration and $2\lfloor(N+$ $1) / 2\rfloor$ tones in the $X$ configuration. An alternative stroboscopic implementation requires only a single auxiliary level, interchanging between the roles of $|e\rangle$ and $|f\rangle$, a single phononic mode, interchanging between being $b$ and $c$, and a single field tone with tunable detuning applied on either the transition $|1\rangle \leftrightarrow|e\rangle$ or $|-\rangle \leftrightarrow|f\rangle$. With $g / 2 \pi \sim 10 \mathrm{kHz}$, typical preparation times are $\tau \sim 30 \mathrm{~ms}$. On such time scales collective dephasing needs to be taken into account, but can be overcome by switching the role of $|0\rangle$ and $|1\rangle$ in the $Z$ pumping for every second ion, thereby preparing $(|0101 \ldots\rangle+|1010 \ldots\rangle) / \sqrt{2}$, which is in a decoherence-free subspace and is equivalent to a GHZ state [13], or by using clock states [55]. Fluctuations of $g$ of $1 \%$ result in a reduction of the fidelity by $0.01-0.1$ for $N=2, \ldots, 8$ qubits, whereas fluctuations of $0.1 \%$ have an effect at the subpercent level. ac Stark shift fluctuations are suppressed since both red- and blue-detuned driving tones (e.g., $\Delta_{ \pm}= \pm \sqrt{n_{1}}$ g) are applied. Heating of the motion would constitute another error on the time scale of the scheme, but this can be made negligible for cryogenic traps [55].

We have shown that dissipative state preparation can be extended to the efficient generation of highly entangled steady states of many particles. We achieve this by engineering complex multiparticle dissipation, which deterministically drives the system into a desired steady state within a time scaling only polynomially with the size of the system. The generic couplings assumed in our approach can be found in a variety of physical systems, such as trapped ions, where the basic ingredients of the scheme have already been demonstrated [34]. As specific examples, we have considered the preparation of highly 
entangled GHZ states, which are paradigmatic multiparticle entangled states, but the developed techniques are applicable to a range of other quantum information tasks. Particularly relevant further possibilities are the construction of quantum error correcting codes [2,3] or the observation of exotic phase transitions [22] induced by multiparticle dissipation.

The research leading to these results has received funding under the European Union's Seventh Framework Programme (FP/2007-2013) through the ERC Grant QIOS (Grant No. 306576) and through SIQS (Grant No. 600645). This work was also supported by the Villum Kann Rasmussen Foundation. F. R. acknowledges helpful conversations with Yiheng Lin and support from the Studienstiftung des deutschen Volkes. D. R. was supported by the Marie Curie Fellowship QUINTYL under Grant No. 298742.

*Present address: Harvard University, Department of Physics, 17 Oxford Street, Cambridge, MA 02138, USA.

[1] M. A. Nielsen and I. L. Chuang, Quantum Computation and Quantum Information (Cambridge University Press, Cambridge, England, 2000).

[2] P. W. Shor, Phys. Rev. A 52, R2493 (1995).

[3] A. M. Steane, Phys. Rev. Lett. 77, 793 (1996).

[4] M. Fleischhauer and M. D. Lukin, Phys. Rev. A 65, 022314 (2002).

[5] V. Giovannetti, S. Lloyd, and L. Maccone, Science 306, 1330 (2004).

[6] G. Toth and I. Apellaniz, J. Phys. A 47, 424006 (2014).

[7] A. Rauschenbeutel et al., Science 288, 2024 (2000).

[8] C. A. Sackett et al., Nature (London) 404, 256 (2000).

[9] F. Schmidt-Kaler, H. Häffner, M. Riebe, S. Gulde, G. P. T. Lancaster, T. Deuschle, C. Becher, C. F. Roos, J. Eschner, and R. Blatt, Nature (London) 422, 408 (2003).

[10] L. DiCarlo, M. D. Reed, L. Sun, B. R. Johnson, J. M. Chow, J. M. Gambetta, L. Frunzio, S. M. Girvin, M. H. Devoret, and R. J. Schoelkopf, Nature (London) 467, 574 (2010); M. Neeley et al., Nature (London) 467, 570 (2010).

[11] D. Leibfried et al., Nature (London) 438, 639 (2005).

[12] H. Häffner et al., Nature (London) 438, 643 (2005).

[13] T. Monz, P. Schindler, J. T. Barreiro, M. Chwalla, D. Nigg, W. A. Coish, M. Harlander, W. Hänsel, M. Hennrich, and R. Blatt, Phys. Rev. Lett. 106, 130506 (2011).

[14] H. M. Wiseman and G. J. Milburn, Quantum Measurement and Control (Cambridge University Press, Cambridge, England, 2009).

[15] V. Bergholm and T. Schulte-Herbrueggen, arXiv:1206.4945.

[16] D. Riste, M. Dukalski, C. A. Watson, G. de Lange, M. J. Tiggelman, Y. M. Blanter, K. W. Lehnert, R. N. Schouten, and L. DiCarlo, Nature (London) 502, 350 (2013).

[17] D. P. DiVincenzo, Fortschr. Phys. 48, 771 (2000).

[18] T. D. Ladd, F. Jelezko, R. Laflamme, Y. Nakamura, C. Monroe, and J. L. O'Brien, Nature (London) 464, 45 (2010).

[19] A. Beige, Phys. Rev. A 69, 012303 (2004).

[20] B. Kraus, H. P. Büchler, S. Diehl, A. Kantian, A. Micheli, and P. Zoller, Phys. Rev. A 78, 042307 (2008).

[21] F. Ticozzi and L. Viola, IEEE Trans. Autom. Control 53, 2048 (2008).
[22] S. Diehl, A. Micheli, A. Kantian, B. Kraus, H. P. Büchler, and P. Zoller, Nat. Phys. 4, 878 (2008).

[23] F. Verstraete, M. M. Wolf, and J. I. Cirac, Nat. Phys. 5, 633 (2009).

[24] J. F. Poyatos, J. I. Cirac, and P. Zoller, Phys. Rev. Lett. 77, 4728 (1996).

[25] M. B. Plenio, S. F. Huelga, A. Beige, and P. L. Knight, Phys. Rev. A 59, 2468 (1999).

[26] A. Beige, D. Braun, and P. L. Knight, New J. Phys. 2, 22 (2000).

[27] X. T. Wang and S. G. Schirmer, arXiv:1005.2114.

[28] M. J. Kastoryano, F. Reiter, and A. S. Sørensen, Phys. Rev. Lett. 106, 090502 (2011).

[29] M. Gullans, T. G. Tiecke, D. E. Chang, J. Feist, J. D. Thompson, J. I. Cirac, P. Zoller, and M. D. Lukin, Phys. Rev. Lett. 109, 235309 (2012).

[30] A. W. Carr and M. Saffman, Phys. Rev. Lett. 111, 033607 (2013).

[31] M. J. A. Schuetz, E. M. Kessler, L. M. K. Vandersypen, J. I. Cirac, and G. Giedke, Phys. Rev. Lett. 111, 246802 (2013).

[32] H. Krauter, C. A. Muschik, K. Jensen, W. Wasilewski, J. M. Petersen, J. I. Cirac, and E. S. Polzik, Phys. Rev. Lett. 107, 080503 (2011).

[33] J. T. Barreiro, M. Müller, P. Schindler, D. Nigg, T. Monz, M. Chwalla, M. Hennrich, C. F. Roos, P. Zoller, and R. Blatt, Nature (London) 470, 486 (2011).

[34] Y. Lin, J. P. Gaebler, F. Reiter, T. R. Tan, R. Bowler, A. S. Sørensen, D. Leibfried, and D. J. Wineland, Nature (London) 504, 415 (2013).

[35] S. Shankar, M. Hatridge, Z. Leghtas, K. M. Sliwa, A. Narla, U. Vool, S. M. Girvin, L. Frunzio, M. Mirrahimi, and M. H. Devoret, Nature (London) 504, 419 (2013).

[36] S. Schneider and G. J. Milburn, Phys. Rev. A 65, 042107 (2002).

[37] G. Vacanti and A. Beige, New J. Phys. 11, 083008 (2009).

[38] H. Weimer, M. Müller, I. Lesanovsky, P. Zoller, and H. P. Büchler, Nat. Phys. 6, 382 (2010).

[39] K. G. H. Vollbrecht, C. A. Muschik, and J. I. Cirac, Phys. Rev. Lett. 107, 120502 (2011).

[40] J. Cho, S. Bose, and M. S. Kim, Phys. Rev. Lett. 106, 020504 (2011).

[41] R. N. Stevenson, J. J. Hope, and A. R. R. Carvalho, Phys. Rev. A 84, 022332 (2011).

[42] M. J. Kastoryano, D. Reeb, and M. M. Wolf, J. Phys. A 45, 075307 (2012).

[43] M. Foss-Feig, A. J. Daley, J. K. Thompson, and A. M. Rey, Phys. Rev. Lett. 109, 230501 (2012).

[44] A. Gonzalez-Tudela and D. Porras, Phys. Rev. Lett. 110, 080502 (2013).

[45] C. Cormick, A. Bermudez, S. F Huelga, and M. B Plenio, New J. Phys. 15, 073027 (2013).

[46] M. Höning, D. Muth, D. Petrosyan, and M. Fleischhauer, Phys. Rev. A 87, 023401 (2013).

[47] S. K. Lee, J. Cho, and K. S. Choi, New J. Phys. 17, 113053 (2015).

[48] F. Ticozzi and L. Viola, Quantum Inf. Comput. 14, 0265 (2014).

[49] D. D. Bhaktavatsala Rao and K. Mølmer, Phys. Rev. A 90, 062319 (2014).

[50] H. Pichler, T. Ramos, A. J. Daley, and P. Zoller, Phys. Rev. A 91, 042116 (2015). 
[51] T. E. Lee, F. Reiter, and N. Moiseyev, Phys. Rev. Lett. 113, 250401 (2014).

[52] W. Happer, Rev. Mod. Phys. 44, 169 (1972).

[53] See Supplemental Material at http://link.aps.org/ supplemental/10.1103/PhysRevLett.117.040501 for the detailed analysis.
[54] F. Reiter and A. S. Sørensen, Phys. Rev. A 85, 032111 (2012).

[55] T. P. Harty, D. T. C. Allcock, C. J. Ballance, L. Guidoni, H. A. Janacek, N. M. Linke, D. N. Stacey, and D. M. Lucas, Phys. Rev. Lett. 113, 220501 (2014). 